\title{
ORIGINAL
}

\section{PREVALENCIA DE INFECCIÓN TUBERCULOSA EN LA POBLACIÓN DE INMIGRANTES EN CEUTA, ESPAÑA}

María Teresa Fernández Sanfrancisco (1), Jacobo Díaz Portillo, José María Sánchez Romero (2), Aitana Pérez Fernández (3) y Juan Vadillo Andrade (1)

(1) Hospital Insalud de Ceuta.

(2) Consejería de Sanidad de la Ciudad Autónoma de Ceuta.

(3) Universidad de Zaragoza.

\section{RESUMEN}

Fundamento: La tuberculosis es la enfermedad infecciosa más extendida en el mundo. Los objetivos de este estudio fueron conocer la prevalencia de infección tuberculosa en los inmigrantes que durante 1998 estuvieron en el campamento de Calamocarro en Ceuta y analizar si existen diferencias según país o región geográfica de procedencia.

Métodos: Estudio descriptivo transversal sobre la población total de inmigrantes en el campamento de Calamocarro en 1998. Se practicó la intradermorreacc ión según la técnica de Mantoux según protocolo ya establecido, a los 2.223 inmigrantes (1979 varones y 244 mujeres) procedentes de distintos países africanos (Nigeria, Mali, Guinea Bissau, Camerún, Ghana, Sierra Leona, Rep Dem Congo, y Liberia, principalmente) en el campamento de refugiados de Calamocarro de Ceuta. Los resultados se presentan para tres puntos de corte de la induración $<5 \mathrm{~mm}$; entre 5 y $10 \mathrm{~mm}$; e igual o superior a $10 \mathrm{~mm}$.

Resultados: Los inmigrantes procedían de 36 países africanos, el $89,0 \%$ eran varones y el $11,0 \%$ mujeres $(p<0,001)$ con una edad media de $24,9 \pm 4,3$ y $23,4 \pm 4,1$ años respectivamente. El $32,6 \%$ presentaron una respuesta al test de Mantoux igual o superior a $10 \mathrm{~mm}, 33,2 \%$ en varones y $27,9 \%$ en mujeres $(p=0,09)$. Sólo hubo un $1,1 \%$ con induración 5-10 $\mathrm{mm}$ de probable origen vacunal. Por zonas geográficas, las tasas más altas se presentaron en inmigrantes procedentes de la República Democrática del Congo $(65,1 \%)$ y de Camerún $(48,4 \%)$, seguidos a distancia por Nigeria $(34,0 \%)$, Liberia $(32,7 \%)$, Mauritania $(29,1 \%)$, Sierra Leona $(28,8 \%)$, Costa de Marfil $(27,8 \%)$, Guinea Bissau $(27,4 \%)$, Ghana $(26,3 \%)$, Argelia $(25,6 \%)$, Mali $(24,1 \%)$ y R.Guinea $(20,9 \%)$ $(\mathrm{p}<0,0001)$.

Conclusiones: La población inmigrante procedente de países centroafricanos presenta una alta prevalencia de infección tuberculosa, constituyendo un grupo de riesgo de padecer la enfermedad. Por tanto, es fundamental la implantación de programas específicos de búsqueda activa de infección tuberculosa durante su estancia en nuestra ciudad, aprovechando su concentración a la entrada en nuestro país, antes de su emigración definitiva a localidades peninsulares y posterior dilución demográfica en la población de acogida.

Palabras clave: Tuberculosis. Inmigrantes. Prevalencia. Prevención.

Correspondencia:

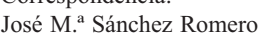

$\mathrm{C} /$ Independencia, $1.3{ }^{\circ} \mathrm{B}$

51001 Ceuta

\section{ABSTRACT}

\section{Tuberculosis Infection Prevalence among the Immigrant Population in Ceuta, Spain}

Background: The immigrant populations from sub-Saharan and Maghreb countries are groups in high risk of contracting tuberculosis. An analysis is made of the prevalence of tuberculosis infection among one group of these immigrants in Ceuta for the purpose of finding possible differences in their incidental behavior according to the country or geographical region from which they came.

Methods: A descriptive cross-sectional study of a sample of 2,223 immigrants (1979 males and 244 females) from different African countries (mainly Nigeria, Mali, Guinea-Bissau, Cameroon, Ghana, Sierra Leona, Democratic Republic of Congo and Liberia) at the Calamocarro refugee camp in Ceuta. A Mantoux intradermic reaction test was performed following the previously-established protocol, the data for three cut-off points being presented.

Results: The immigrants came from 36 African countries, $89.0 \%$ being males and $11.0 \%$ females $(\mathrm{p}<0.001)$, respectively averaging in age from $24.9 \pm 4.3$ to $23.4 \pm 4.1$. A reaction to the Mantoux text of $10 \mathrm{~mm}$ or more was found in $32.6 \%$, that is $33.2 \%$ among males and $27.9 \%$ among females $(\mathrm{p}=0.09)$. Solely $1.1 \%$ showing $5-10 \mathrm{~mm}$ hardening of probable vaccination-related cause. By geographical regions, the highest rates were found among immigrants from the Democratic Republic of Congo $(65.1 \%)$ and Cameroon $(48.4 \%)$, followed far behind by Nigeria $(34.0 \%)$, Liberia $(32.7 \%)$, Mauritania $(29.1 \%)$, Sierra Leona $(28.8 \%)$, Ivory Coast $(27.8 \%)$, Guinea-Bissau $(27.4 \%)$, Ghana $(26.3 \%)$, Algeria $(25.6 \%)$, Mali $(24.1 \%)$ and the Republic of Guinea $(20.9 \%)(\mathrm{p}<0.0001)$.

Conclusions: The immigrant population from central African countries shows a higher prevalence of tuberculosis infection, comprising a group at risk of contracting this disease. Therefore, it is of fundamental importance to implement specific programs to actively detect tuberculosis infection during their stay in our city, taking advantage of their being grouped together upon entry into our country, prior to their final emigration to localities throughout the mainland and subsequent mixing among the host country population. ion 


\section{INTRODUCCIÓN}

La tuberculosis (TB) es una de las enfermedades infecciosas de mayor importancia en nuestro país y en gran parte del mundo, constituyendo un importante problema de salud pública, razón por la cual la Organización Mundial de la Salud (OMS) la declaró emergencia global en $1993^{1}$.

Se estima que se producen en torno a 8 millones de casos nuevos anualmente en todo el mundo que unidos a los 12 millones ya existentes arrojan un total de 20 millones de casos, lo que la convierte en la enfermedad infecciosa más prevalente ${ }^{2}$. Los países en vías de desarrollo son los más frecuentemente afectados, aportando más del 95\% de los casos, en especial los localizados en el África Subsahariana, estimándose la incidencia de enfermedad tuberculosa en estos países en 7 millones de personas/año ${ }^{3}$.

Algunos países desarrollados en la década de los 80 experimentaron un importante incremento en la incidencia de la enfermedad. Las causas de este cambio de tendencia fueron la persistencia de bolsas de pobreza y marginalidad, la eclosión del VIH y el descuido o abandono de los programas antituberculosos. En los países desarrollados, las minorías étnicas procedentes de países con alta prevalencia constituyen un núcleo de persistencia de la TB, aportando entre el $23-32 \%$ de los casos de TB activa ${ }^{5,6}$. En América del Norte más de la tercera parte de los casos diagnosticados proceden de inmigrantes $^{7}$, representando en algunos estados de Canadá hasta el 71\% de los casos diagnosticados ${ }^{8}$. En Europa occidental la inmigración es un fenómeno social que supone actualmente el 3\% de la población, aportando, según el país, del $7 \%$ al $68 \%$ de los enfermos ${ }^{5,9,10}$. En algunos países europeos (Suiza, Holanda, Dinamarca) el aumento de nuevos casos de TB en inmigrantes procedentes de países en vías de desarrollo ha contribuido a desequilibrar la tendencia decreciente de la enfermedad que venía observándose en los últimos años ${ }^{11}$.
La TB en España presenta características que la diferencian de los países de su entorno socioeconómico. En comparación con otros países europeos, la contribución del inmigrante a la epidemiología de la TB en España podríamos calificarla de moderada o baja, no pudiendo decir que los inmigrantes hagan una contribución importante a la magnitud del problema de la TB en nuestro país, aunque en los últimos años ha aumentado el porcentaje de extranjeros entre los casos declarados de tuberculosis en España. Por lo que de persistir el aumento del flujo migratorio sin las medidas preventivas necesarias $^{12,13}$,sería probable que se repitiese lo sucedido en la mayoría de países desarro1lados 5 .

España se ha convertido en los últimos años en un país receptor de inmigrantes procedentes de países de escasos recursos y delicada situación social y política, que buscan mejorar sus condiciones de vida. La ciudad de Ceuta, por su especial situación geográfica, constituye actualmente un lugar de paso obligatorio para un amplio colectivo de inmigrantes procedentes de países en vías de desarrollo, mayoritariamente del norte y del centro de África, cuyo destino final son diversas localidades peninsulares. El notable incremento que ha sufrido esta inmigración en los últimos cinco años y su procedencia mayoritaria del Africa Subsahariana despiertan inquietud social y corrientes de opinión muy diversas, incluidas claras actitudes de xenofobia que equivocadamente relacionan la inmigración con el fracaso en el control de las enfermedades infecciosas ${ }^{14}$.

El campamento de refugiados de Calamocarro acogió temporalmente a los inmigrantes antes de su posterior tránsito a la península. Dicho campamento, con una extensión aproximada de 20.000 metros cuadrados, tenía instaladas una serie de grandes tiendas de campaña donde se dormía en literas individuales. En la zona destinada a los servicios estaban las duchas y aseos; las comidas se servían en un pabellón diferente en el que había un salón de televisión, y que estaba 
próximo a un consultorio médico prefabricado que funcionaba durante la mañana.

El objetivo de este trabajo fue determinar la prevalencia de infección tuberculosa en los inmigrantes africanos recién llegados al campamento de Calamocarro, Ceuta, durante 1998 , y ver si existían diferencias según su país o región geográfica de procedencia.

\section{SUJETOS Y MÉTODOS}

Se realizó un estudio descriptivo observacional de corte transversal sobre los 2.223 inmigrantes (1.979 varones y 244 mujeres) que estuvieron en Calamocarro durante el año 1998, los cuales procedían de distintos países africanos (Nigeria, Mali, Guinea Bissau, Camerún, Ghana, Sierra Leona, República Democrática del Congo, y Liberia, principalmente). Tras su traslado hasta el citado Campamento, agentes de la Cruz Roja, les atendían, asesoraban y prestaban asistencia médica. El idioma en el que se entendían los inmigrantes con los agentes de la Cruz Roja era el inglés o el francés.

El Área Funcional de Sanidad realiza sistemáticamente un reconocimiento médico a todos los inmigrantes que llegan a nuestra ciudad. El reconocimiento incluye: test tuberculínico, analítica general (hemograma completo, marcadores del VHB y serología de la sífilis), radiografía de tórax si procede, examen coproparasitológico y otras pruebas complementarias si fuese preciso en función de los resultados obtenidos con las primeras pruebas. Se practicó la intradermorreacción mediante la técnica de Mantoux, siguiendo las recomendaciones del Consenso Nacional para el Control de la TB en España ${ }^{15}$, inyectando 2 UT de tuberculina PPD RT23 por vía intradérmica, y para su lectura se empleó la técnica de Sokal ${ }^{16}$. Se realizó baciloscopia y cultivo de esputo en medio de Löwenstein a los sujetos con PPD de más de $10 \mathrm{~mm}$ de induración y signos radiológicos sugestivos o sintomatología específica de TB.
Se registraron los datos de los inmigrantes, incluyendo los de su filiación, país de origen y fecha de nacimiento, así como el resultado de todas las pruebas realizadas.

El análisis estadístico se ha efectuado mediante el programa informático Epiinfo 2.000 versión 1.05 (www.cdc.gob/epiinfo). Se realizó la estadística descriptiva de las variables mediante distribución de frecuencias, medidas de tendencia central, desviación estándar y cálculo de los intervalos de confianza al 95\% (IC del 95\%). Empleamos el test de chi $^{2}$ para comparación de porcentajes, y la prueba de la t de Student para variables cuantitativas. La evaluación de las tendencias se realizó con la prueba de chi $^{2}$ de tendencia lineal. Se han analizado las distintas prevalencias de positividad según la zona geográfica de procedencia, calculando el intervalo de confianza según la prueba de Cornfield. Los datos de prevalencia se representan como el porcentaje de sujetos con reacción positiva para 3 puntos de corte de la reacción de Mantoux $(<5 \mathrm{~mm},<10 \mathrm{~mm}$ y $<15 \mathrm{~mm}$ ), y su correspondiente intervalo de confianza al 95\% (IC del 95\%).

\section{RESULTADOS}

De los 2.223 inmigrantes que pasaron por el campamento de Calamocarro en 1998, $1.979(89,0 \%)$ eran varones y $244(11,0 \%)$ mujeres $(\mathrm{p}<0,001)$, con una edad media de $25,4 \pm 4,6$ y $23,3 \pm 5,0$ años respectivamente $(p<0,0001)$. Los inmigrantes eran originarios de 36 países africanos, principalmente subsaharianos, localizados en el golfo de Guinea, en la zona central del continente. El país de procedencia más representado fue Nigeria con 597 individuos $(27,4 \%)$ (tabla 1).

En la tabla 2 se presentan los datos de la prueba del Mantoux (PPD) para el total de individuos examinados, agrupados según sexo, edad y país de origen. De los 2.216 PPD leídos (sobre 2.223 realizados) 722 $(32,6 \%)$ presentaron una respuesta al test de 
Tabla 1

Características sociodemográficas de la población inmigrante en Ceuta en 1998

\begin{tabular}{|lcc|}
\hline Variables & Número & Porcentaje \\
\hline Sexo & & \\
Varón & 1979 & 89,0 \\
Mujer & 244 & 11,0 \\
Grupo etario & & \\
14-24 años & 942 & 54,8 \\
25-34 años & 724 & 42,1 \\
$>35$ años & 53 & 3,1 \\
& & \\
País de origen & & \\
Argelia & 133 & 6,1 \\
Costa de Marfil & 18 & 0,8 \\
Camerún & 128 & 5,9 \\
Gambia & 107 & 4,9 \\
Ghana & 95 & 4,4 \\
Guinea Bissau & 186 & 8,6 \\
Liberia & 105 & 4,9 \\
Mali & 291 & 13,4 \\
Mauritania & 55 & 2,5 \\
Nigeria & 597 & 27,4 \\
R.D. Congo & 146 & 6,7 \\
R. Guinea & 45 & 2,1 \\
Sierra Leona & 177 & 8,2 \\
\hline
\end{tabular}

Mantoux igual o superior a $10 \mathrm{~mm}$, de los cuales $494(22,2 \%)$ presentaron una induración mayor a $15 \mathrm{~mm}$. Sólo 24 casos $(1,1 \%)$ presentaban induración entre $5-10 \mathrm{~mm}$. Con independencia del punto de corte seleccionado, se observó mayor positividad en el sexo masculino que en el femenino, y la positividad también presentó una ligera tendencia a aumentar con la edad $(\mathrm{p}=0,07)$. La edad media de los sujetos con PPD positivo fue de 25,5 $\pm 4,4$ años, con un diámetro medio de induración de $18,7 \pm 6,2 \mathrm{~mm}$. No se valoró el estado vacunal de los sujetos ni se les realizó pruebas de detección del VIH a nivel colectivo.

Por zonas geográficas, las tasas más altas se presentaron en inmigrantes procedentes de la República Democrática del Congo $(65,1 \%)$ y del Camerún $(48,4 \%)$, seguidos a distancia por Nigeria $(34,0 \%)$, Liberia $(32,7 \%)$, Mauritania $(29,1 \%)$, Sierra Leona
Tabla 2

Prevalencia de infección tuberculosa de la población inmigrante en Ceuta en 1998 por sexo, edad y país de procedencia

\begin{tabular}{|c|c|c|}
\hline Variables & Número de casos & Porcentaje \\
\hline \multicolumn{3}{|l|}{ Sexo } \\
\hline Varón & 654 & 33,2 \\
\hline Mujer & 68 & 27,9 \\
\hline $\begin{array}{l}\text { Diferencias entre } \\
\text { sexos }\end{array}$ & \multicolumn{2}{|c|}{ Test de $\mathrm{chi}^{2}=2,8 ; \mathrm{p}=0,09$} \\
\hline \multicolumn{3}{|l|}{ Grupo etario } \\
\hline $14-24$ & 221 & 28,2 \\
\hline $25-34$ & 242 & 33,5 \\
\hline$>35$ & 15 & 27,8 \\
\hline $\begin{array}{l}\text { Diferencias por } \\
\text { grupo de edad }\end{array}$ & \multicolumn{2}{|c|}{$\begin{array}{l}\text { Test de } \text { chi }^{2} \text { de tendencia }=5,2 \\
\qquad p=0,07\end{array}$} \\
\hline \multicolumn{3}{|l|}{ País de origen } \\
\hline Argelia & 34 & 25,6 \\
\hline Camerún & 62 & 48,4 \\
\hline Costa de Marfil & 5 & 27,8 \\
\hline Gambia & 12 & 11,2 \\
\hline Ghana & 25 & 26,3 \\
\hline Guinea Bissau & 51 & 27,4 \\
\hline Liberia & 34 & 32,7 \\
\hline Mali & 69 & 24,1 \\
\hline Mauritania & 16 & 29,1 \\
\hline Nigeria & 202 & 34,0 \\
\hline R. D. Congo & 95 & 65,1 \\
\hline Rep. Guinea & 9 & 20,9 \\
\hline Sierra Leona & 51 & 28,8 \\
\hline $\begin{array}{l}\text { Diferencias por } \\
\text { país de origen }\end{array}$ & Test de $\mathrm{chi}^{2}=165$ & $<0.00001(*)$ \\
\hline
\end{tabular}

$(*)$ Se aprecian diferencias estadísticamente significativas en la prevalencia de infección tuberculosa en los distintos países.

(28,8\%), Costa de Marfil (27,8\%), Guinea Bissau (27,4\%), Ghana (26,3\%), Argelia $(25,6 \%)$, Mali $(24,1 \%)$ y R.Guinea $(20,9 \%)$ $(\mathrm{p}<0,0001)$ (tabla 2).

No se descartaron previamente los casos de tuberculosis anterior. No obstante estos sujetos eran captados en los estudios que se realizaban posteriormente. En dichos estudios complementarios se detectaron 19 casos con imágenes radiológicas pulmonares sugestivas de enfermedad tuberculosa, siendo finalmente hallados dos casos de bacilosocpia positiva, a los que se les prescribió 
tratamiento con pauta 6RHZ. Asimismo aquellos que eran diagnosticados de infección tuberculosa latente eran tratados con una quimioprofilaxis de isoniazida 300 $\mathrm{mg} / 24$ horas en ayunas durante seis meses.

\section{DISCUSIÓN}

Existen muy pocos datos sobre el estado de salud de los inmigrantes antes de su asentamiento definitivo en nuestro país, ya que los datos publicados se refieren a poblaciones de inmigrantes ya residentes ${ }^{17-19}$. En el campamento de refugiados de Calamocarro encontramos generalmente personas jóvenes, mayoritariamente varones, originarios de diversos países subsaharianos, principalmente centroafricanos, con una situación de ilegalidad, inestabilidad social, religión predominantemente musulmana y dificultad de comunicación en español.

En la prevención y control de la $\mathrm{TB}$ es fundamental identificar en cada área o grupo poblacional a aquellos individuos con riesgo de infección y establecer protocolos y modos de actuación sanitarios que permitan reducir la incidencia de la enfermedad, por lo que sería beneficioso reglamentar un estudio previo de salud a los inmigrantes que lleguen a nuestro país en el que se incluyese la prueba de la tuberculina, como ocurre en Francia y USA, países con una alta tasa de inmigración y con programas de prevención y control de TB. La tasa de incidencia de TB en España, 38,51/100.000, es la más alta de Europa Occidental (con excepción de Portugal) y más alta que en América del Norte, lo que nos califica como un país con TB común ${ }^{20}$. Sin embargo la prevalencia en los países del Norte de África es notablemente superior, diferencia que hace de los inmigrantes de origen norteafricano un importante grupo de riesgo para la enfermedad e indica la aplicación sistemática de programas encaminados a la búsqueda y control de $\mathrm{TB}$, específicamente dirigidos a entre otros colectivos al de inmigrantes ${ }^{9,12,21}$.
La prueba de la tuberculina es, por el momento, el único método para identificar la infección tuberculosa, y es una herramienta básica en el diagnóstico y en la investigación epidemiológica de la TB. Un resultado positivo en una persona indica que ha estado en contacto con el bacilo, por lo que la tasa de individuos con resultados positivos para el test o prevalencia de la infección tuberculosa en una comunidad es un indicador objetivo del número de personas que han tenido dicho contacto ${ }^{22}$. Hemos tomado como punto de corte de referencia la medida de 10 $\mathrm{mm}$, ya que en otros estudios no se han encontrado diferencias significativas en la respuesta a la tuberculina entre vacunados y no vacunados cuando ésta es mayor de 10 $\mathrm{mm}^{23}$. Destacamos la elevada prevalencia de reacción positiva a la tuberculina en la población inmigrante recién llegada a nuestra ciudad, siendo ésta comparable a las descritas por otros autores en grupos de refugiados o inmigrantes en otros países ${ }^{8,24-27}$. Nuestros valores son similares a los hallados entre los inmigrantes procedentes de Oriente Medio y norte de África en un estudio realizado sobre una población de 1.489 individuos recién llegados a Barcelona ${ }^{23}$, en el que considerando como prueba positiva una reacción igual o superior a $10 \mathrm{~mm}$ encuentran una prevalencia del $34 \%$. Sin embargo, la prevalencia global en nuestro colectivo fue claramente inferior al $72,5 \%$ descrito en un grupo de 80 inmigrantes magrebíes (principalmente marroquíes) residentes en Barcelona ${ }^{28}$. Esta diferencia podría ser justificada por la distinta procedencia de los sujetos estudiados, ya que la población de nuestro estudio es mayoritariamente centroafricana.

La frecuencia de resultados PPD positivos mostró importantes diferencias según el país de procedencia; con una mayor tasa de infección en inmigrantes procedentes de la República Democrática del Congo $(65,1 \%)$ y Camerún $(48,4 \%)$, que tenían prevalencias notablemente superiores a la media. Este alto nivel justifica la realización sistemática de la intradermorreacción de Mantoux como 
cribado de la TB en la población de inmigrantes que actualmente reside en nuestra área sanitaria, al objeto de poder tomar medidas sanitarias en los casos positivos, estudio radiológico de tórax y prescripción de isoniazidas en caso de tratarse una infección TB latente. Como el tiempo de estancia media en el Campamento era de tres a seis meses se enviaba a la Asamblea local de la Cruz Roja (que se encargaba de recopilar su documentación y viaje) un informe sanitario con la recomendación de continuar hasta la finalización el tratamiento prescrito, debiendo ser éste supervisado y controlado clínicamente y por medio de marcadores hepáticos, por el servicio médico de acogida en la ciudad de destino una vez que los inmigrantes se marchaban de Ceuta.

Los dos casos de TB pulmonar diagnosticados en este colectivo nos indicarían una tasa superior a la de la población española y del resto de países de la UE, pero muy cercana a la de los países centroafricanos. Podríamos admitir que la situación del inmigrante respecto a la TB podría ser un reflejo directo de la existente en su país ${ }^{5}$, aunque hay que tener en cuenta que las tasas estimadas en los países de origen están condicionadas por la desnutrición y por otras enfermedades debilitantes que hacen mella en la población, mientras que los inmigrantes constituyen la parte más sana, joven y saludable del país en cuestión, por lo que la extrapolación es difícil y habría que asumir que tiene una posibilidad inferior de padecer $\mathrm{TB}^{29}$.

Por otro lado, tenemos que reflexionar sobre la posibilidad de que estos inmigrantes, una vez situados en las localidades peninsulares de destino final, no vayan a acudir a los centros sanitarios ante síntomas sugestivos de tuberculosis por considerar que de este modo corren el riesgo de que se les identifique y expulse del país, lo que aumentaría la tasa de enfermos no tratados en la comunidad de acogida que podrían diseminar la infección tuberculosa ${ }^{29}$. Esta circunstancia justificaría la importancia del control de la infección tuberculosa en nuestra ciudad, durante su estancia en el campamento de refugiados, donde deben residir mientras se regulariza su situación de inmigrante, condición ésta necesaria e imprescindible para su posterior traslado a localidades peninsulares, y posterior dilución demográfica en la población de acogida. Por otro lado, las peculiares características de este grupo de ries$\mathrm{go}^{30}$ (bajo nivel socioeconómico y cultural, desconocimiento del idioma español, desarraigo sociocultural) podrían actuar dificultando el diagnóstico y el tratamiento en los enfermos, pudiendo potenciar la transmisión de la infección en dicho colectivo ${ }^{28}$. Todos estos factores podrían estar justificando el bajo grado de cumplimiento de la quimioprofilaxis y el alto índice de abandono precoz que describen algunos autores en la evaluación de programas específicos de prevención y control dirigidos a estos colec$\operatorname{tivos}^{23-31}$.

Pensamos que, por su condición especial de marginación social y breve estancia en nuestra cuidad (siempre inferior a un año), el inmigrante africano no va a suponer ninguna influencia en la situación epidemiológica de la TB en Ceuta. Sin embargo, posteriormente en las poblaciones peninsulares de acogida, podrían convertirse en un grupo con riesgo de servir de amplificadores epidemiológicos de diferentes agentes etiológicos transmisibles, entre los cuales destaca la infección tuberculosa. Por todo ello, recomendamos la puesta en marcha en sus lugares de destino de programas de seguimiento para asegurar el cumplimiento del tratamiento y control de la posible aparición de resistencias microbianas de Mycobacterium tuberculosis.

\section{AGRADECIMIENTOS}

Al Delegado del Gobierno en Ceuta, el poner a nuestra disposición los datos primarios para la elaboración de este trabajo. 


\section{BIBLIOGRAFÍA}

1. TB. A Global Emergency. WHO Report on the TB Epidemic. WHO/TB/94.177.

2. Dye C, Scheele S, Dolin O, Pathania V, Raviglione M. For the WHO Global Surveillance and Monitoring Project. Global Burden of Tuberculosis. Estimated Incidence, Prevalence, and Mortality by Country. JAMA 1999; 282: 677-686.

3. CDC. Tuberculosis in developing countries. MMWR 1990; 33: 561-569.

4. Piédrola Gil G. En: Medicina Preventiva y Salud Pública. Madrid: 2.001: 507.

5. Clancy L, Rieder HL, Enarson DA, Spinaci S. Tuberculosis elimination in the countries of $\mathrm{Eu}-$ rope and other industrialized countries. Eur Respir J 1991; 4: 1.288-1.295.

6. De March Ayuela P. Situación actual de la tuberculosis en España. Med Clin (Barc.) 1992; 98: 24-31.

7. CDC. Recommendations for Prevention and Control of Tuberculosis Among Foreing-Born Persons. MMWR 1998; 47: 1-26.

8. Robert L, Cowie MD, Jill W, Sharpe BN. Tuberculosis among immigrants: interval from arrival in Canada to diagnosis. CMAJ 1998; 158: 4599-602.

9. Hardie RM, Watson JM. Screening immigrants at risk of tuberculosis. BMJ 1993; 307: 1539-1540.

10. Word Health Organization. Global Tuberculosis Control. WHO Report 2000. Ginebra: World Health Organization; 2000.

11. CDC. Tuberculosis Western Europe, 1974-1991. MMWR 1993; 32: 628-631.

12. De March Ayuela P. Situación actual de la tuberculosis en España. Med Clin (Barc.) 1991; 97 : 463-472.

13. Burgos M, Asensio O, De Mateo Ontañon. Situación actual y evolución de la tuberculosis en España. Bol Epidemiol Sem 1996; 4: 156-160.

14. Caminero JA. Medidas básicas para el control de la tuberculosis en una comunidad. Med Clin (Barc) 1994; 102: 67-73.

15. Ministerio de Sanidad y Consumo. Fondo de Investigación Sanitaria. Consenso Nacional para el Control de la Tuberculosis en España. Med Clin (Barc) 1992; 98: 24-31.
16. Sokal JE. Measurement of delayed skin-test responses. N Engl J Med 1975; 293: 501-2.

17. Roca C, Balanzó X, Fernández JL, Pujol E, Corachan M. Caracterización demográfica, motivos de consulta y morbilidad prevalente en la comunidad de inmigrantes africanos de la comarca del Maresme. Med Clin (Barc) 1999; 111: 215-7.

18. Lacalle M, Gil G, Sagardui E, González E, Martínez R, Orden B. Resultados de la aplicación de un examen de salud en población emigrante. Aten Primaria 2000; 9: 78-86.

19. Esteban M, Peña M. Motivos de consulta y características demográficas de una comunidad de inmigrantes «sin papeles» en el distrito de Usera-Villaverde (Madrid) Aten Primaria 2001; 1 : $25-8$.

20. Grupo de Trabajo del Proyecto Multicéntrico de Investigación sobre Tuberculosis (PMIT). La Tuberculosis en España: resultados del Proyecto Multicentrico de Investigación sobre Tuberculosis 1999.

21. Consejería de Sanidad y Servicios Sociales. Encuesta de Tuberculina en la Comunidad de Madrid. Madrid: Consejería de Sanidad y Servicios Sociales; 1995. Documento Técnico de Salud Pública núm 28.

22. Alcaide J, Altet J, Canela J. Epidemiología de la tuberculosis. An Esp Pediatr 2000; 53: 449-457.

23. Durán E, Cabezos J, Ros M, Terre M, Zarzuela F, Bada JL. Tuberculosis en inmigrantes recién llegados a Barcelona. Med Clin (Barc) 1996; 106: $525-8$.

24. Godue ChB, Gogging P, Giorkos TW. L'allergie tuberculinique chez les revendicateurs du statut de refugié nouvellement arrivés a Canada. Can Med Assoc J 1998; 139: 41-4.

25. Peréz-Stable EJ, Slutkin G, Paz EA, Hopewell PC. Tuberculin reactivity in United States and Foreing-Born Latinos: results of a Community Based Screening Program. Am J Public Health 1986; 76: 643-6.

26. Berger SA, Schwartz T, Michaeli D. Infectious disease among ethiopian immigrants in Israel. Arch Intern Med 1989; 149:117-9.

27. De Riemer K, Chin DP, Schecter GF, Reingold AL. Tuberculosis among immigrants and refugees. Arch Intern Med 1998; 158: 753-760. 
28. Rivas FPJ, Nácher M, Corrillero J, García-Herreros T. Prevalencia de la infección tuberculosa entre los inmigrantes magrebíes. Med Clin (Barc) 2000; 114: 245-249.

29. Caminero JA, Rodríguez F, Cuyás J, Cabrera P. La inmigración en Canarias y su posible influencia sobre la frecuencia de tuberculosis. Med Clin (Barc) 2001; 116: 556-7.
30. Palomo M, Rodríguez C, Ayerbe T. Un estudio de contactos de tuberculosis en inmigrantes marroquíes. Rev Esp Salud Pública 1996; 70: 345-9.

31. Rivas FPJ, Nácher M, Corrillero J, Velez S, Huerta L. Resultados de un programa de búsqueda activa de tuberculosis en inmigrantes magrebíes: aceptabilidad y adhesión. Aten Primaria 1999; 7: 411-6. 